\title{
Investigating non-response bias in a survey of disablement in the community: implications for survey methodology
}

\author{
Alan Tennant, Elizabeth M Badley
}

\begin{abstract}
Study objective-The aim was to investigate the pattern of age specific nonresponse bias in a two phase survey of disablement in the community. It seeks to examine patterns of response in different age groups to a household based postal questionnaire, and the implication of such trends for the estimation of prevalence of reported dependence. It also looks at the effect that the readiness to respond during the first phase postal questionnaire had on participation in the interview based second phase of the study.
\end{abstract}

Design and setting-A two stage survey of disablement in the population was undertaken. A first phase postal questionnaire was sent to 25168 households in Calderdale, West Yorkshire, England, to ascertain the prevalence of physical disability. The second phase comprised in depth interviews with a sample of individuals identified in the first phase as being disabled.

Respondents-A total of 21889 postal questionnaires were returned (87\%) representing households containing 42826 people aged 16 years and over. A disproportionately stratified random sample of 950 respondents reporting disability was taken for the second phase. Of these 891 were still available, and 838 (94\%) were interviewed.

Measurements and main results-A study of the timing of response to a postal questionnaire showed that patterns differed for different age groups. The estimated prevalence of those aged 65 years and over who were dependent was steady over time whereas for those in the 16-64 age range the estimated prevalence fell as the survey progressed, indicating a tendency for those who were dependent to respond sooner. Examination of the relationship of responses at phase 1 and phase 2 showed that response to invitation to interview was much less in those who had responded later, and presumably more reluctantly, in the first phase.

Conclusions-These findings raise questions about how different patterns of response might be indicative of bias which could differentially affect final age specific prevalence estimates. They also have methodological implications for the follow up of reluctant responders both to increase the response rate and to secure cooperation in the second phase of a two phase survey.
Respondents who fail to reply to postal questionnaires or refuse an interview are always a cause of concern. Incomplete response introduces uncertainty as to the accuracy of the findings, ${ }^{1}$ hence the desire to get as high a response rate as possible. Given that complete ascertainment is rarely, if ever, feasible, questions arise as to how one might gain some kind of insight into the nature of non-response.

The emergence of different age specific response patterns in a two stage population survey of the physically disabled prompted us to look further at the question of response bias, particularly as we found that reports from other surveys of disablement in the population differed in their treatment of non-response. ${ }^{23}$

\section{Methods}

The survey was carried out to help plan the provision of services for those in the population of Calderdale in West Yorkshire, England, who were very severely physically disabled and likely to be on the margins of institutional care. ${ }^{4}$ A primary focus of the survey was the so called younger physically disabled population, those aged 16-64 years, although the survey was extended to look at those aged 65 years and older who were disabled by rheumatic conditions. A two stage methodology was employed, similar to that used in other surveys of disablement in the population. ${ }^{5}$ The first stage consisted of a postal questionnaire to every third household in the area, using the Domestic Rating List as the sampling frame. The sample size was determined by the need to provide meaningful analysis for a rare group in the population, namely those very severely physically disabled aged 16-64 years, based on an estimate by Harris of 12 per 10000 people. $^{3}$ The second stage was an in depth personal interview with a stratified random sample of those ascertained to be disabled in phase 1 of the survey. This paper is primarily concerned with response in the phase 1 screening survey.

The four page screening questionnaire used in phase 1 elicited demographic characteristics of the household, and information about individuals within the household who experienced difficulties or dependence in various activities of daily living.

The main target of the survey was those who were very severely disabled and who might be expected to have high dependency needs. Therefore the principal entry criteria for the second stage of the survey was set at the level of being dependent on others; specifically requiring help to get in or out of bed, with dressing, to get to and use the toilet, or to get out of the house. This latter condition sets a relatively low threshold for 
entry and is consistent with an approach to screening for rare groups in populations which minimises false negatives and produces a manageable group for further investigation. ${ }^{67}$ This paper concerns the ascertainment of this dependent group in the population, and considers non-response in terms of non-return of the questionnaire or refusal of interview.

Questionnaires were sent to 25168 occupied dwellings in Calderdale and 21889 were returned, a response rate of $87 \%$. This was achieved by the original post (1st wave, $57 \%$ response), two further postal follow ups (2nd and 3 rd waves, taking the response to $73 \%$ and $81 \%$ respectively), the latter including a small personal call back, and a final postal follow up (the 4th wave). The final postal follow up was encouraged by findings from the personal call back which indicated that one quarter of respondents still outstanding would return another questionnaire which was left with them or put through their door. It was carried out in order to minimise potential bias due to a "low" response rate which, at the end of the third wave, stood at $81 \%$.

Table I shows the number of people responding within each major age group at each stage of the study. The demographic data from households responding at phase 1 were used as the denominator to calculate the estimated rate per thousand dependent people in each age group. The cumulative rate was plotted in order to monitor trends in ascertained prevalence as the survey progressed.

The sample for the phase 2 in depth interviews comprised 950 individuals. The overall response in this phase was $88 \%$, which represented $94 \%$ of

Table I Age and sex structure of the total survey population and number and proportion who live alone.

\begin{tabular}{|c|c|c|c|c|c|c|}
\hline \multirow[b]{2}{*}{$\begin{array}{l}\text { Age group } \\
\text { (years) }\end{array}$} & \multicolumn{2}{|c|}{ All persons } & \multicolumn{2}{|l|}{ Males } & \multicolumn{2}{|c|}{ Females } \\
\hline & $\begin{array}{l}\text { Total } \\
n\end{array}$ & $\begin{array}{l}\text { Lives alone } \\
n(\%)\end{array}$ & $\begin{array}{l}\text { Total } \\
n\end{array}$ & $\begin{array}{l}\text { Lives alone } \\
n(\%)\end{array}$ & $\begin{array}{l}\text { Total } \\
n\end{array}$ & $\begin{array}{l}\text { Lives alone } \\
n(\%)\end{array}$ \\
\hline $\begin{array}{l}16-34 \\
35-54 \\
55-64 \\
65-74 \\
\geqslant 75\end{array}$ & $\begin{array}{r}14577 \\
13175 \\
5967 \\
5441 \\
3666\end{array}$ & $\begin{array}{r}951(6.5) \\
953(7 \cdot 2) \\
883(14 \cdot 8) \\
1502(27.6) \\
1899(51.8)\end{array}$ & $\begin{array}{l}7244 \\
6592 \\
2776 \\
2398 \\
1221\end{array}$ & $\begin{array}{ll}376 & (5 \cdot 2) \\
459 & (7 \cdot 0) \\
272 & (9 \cdot 8) \\
386(16 \cdot 1) \\
365(29 \cdot 9)\end{array}$ & $\begin{array}{l}7333 \\
6583 \\
3191 \\
3043 \\
2445\end{array}$ & $\begin{array}{r}575(7 \cdot 8) \\
494(7 \cdot 5) \\
611(19 \cdot 1) \\
1116(36 \cdot 7) \\
1534(62 \cdot 7)\end{array}$ \\
\hline Total & 42826 & $6188(14 \cdot 4)$ & 20231 & $1858 \quad(9 \cdot 2)$ & 22595 & $4330(19 \cdot 2)$ \\
\hline
\end{tabular}

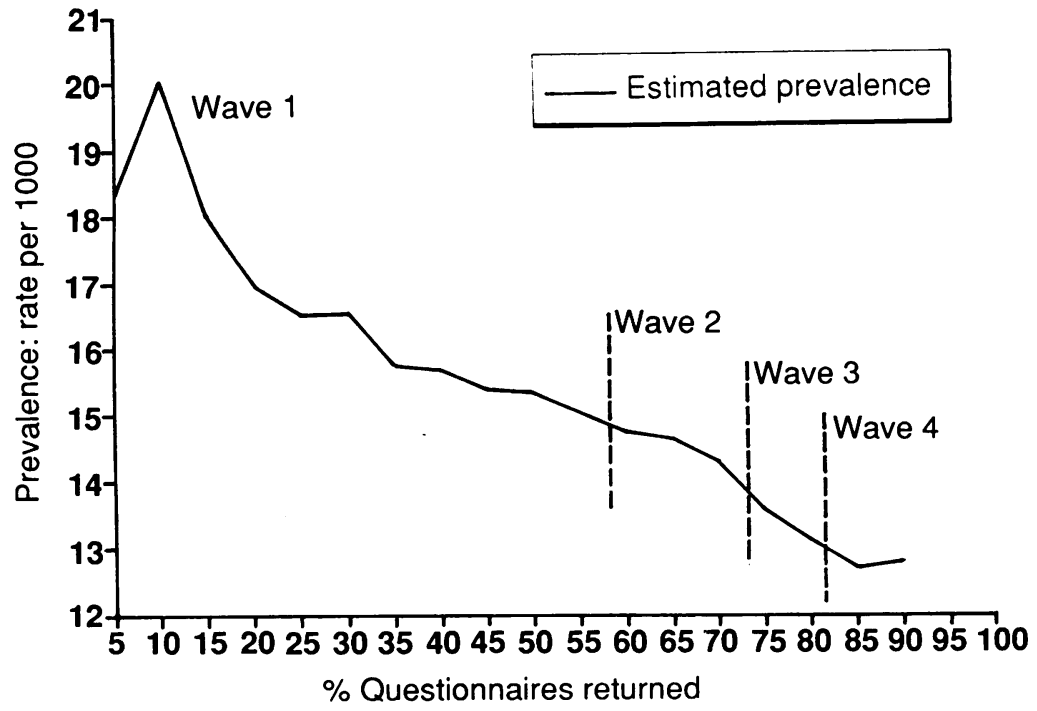

Figure 1 Cumulative rate of dependence (respondents aged 16-64 years) those eligible to be seen (ie, excluding those who had died, entered institutions, or moved away; the average time between the phase 1 screen and interview was three months).

\section{Results}

Figure 1 shows the cumulative estimated prevalence (expressed as rate per 1000 people) for the main target group of the survey, respondents aged 16-64 years who were dependent on others. The four waves of the survey are identified. We think the fluctuation in the earlier part of the survey is a function of taking advantage of the discounts for bulk mailing, where the questionnaire was delivered over a number of days. Consequently responses from different areas of Calderdale were staggered.

Figure 1 shows that the cumulated prevalence fell as a greater proportion of the survey was returned, indicating a tendency for those in this age group who were dependent to reply sooner. At the end of the phase 1 response the final estimated prevalence was $12 \cdot 8$ persons per 1000 population (95\% CI: $11 \cdot 3-14 \cdot 4)^{8}$ aged $16-64$ years who were dependent upon others for one or more of toileting, getting in and out of bed, dressing, and getting in and out of the house.

Figure 2 presents the same information for those aged 65 years and over. After the first few days of erratic response the prevalence of dependent people aged 65 years and over remained similar throughout the survey; in other words there was little difference in prevalence of dependency amongst early (1st wave) and late (4th wave) responders. The final estimated prevalence for this group was $132 \cdot 1$ persons per 1000 population (95\% CI: $123 \cdot 1-140 \cdot 8)$

Investigation showed differing response patterns when the major age groups were further disaggregated. For example, fig 3 shows the prevalence of dependent 16-34 year olds throughout the survey. The response pattern was generally upward, indicating a reluctance of those with dependence in this age group to return the questionnaire. This is of concern as the age group was of particular interest to those involved in planning services for the younger physically disabled. Although small in numbers, it includes individuals with special needs, for instance in negotiating the transfer from school to work. The final estimated prevalence for this 16-34 year old group was 4.2 persons per 1000 population $(95 \%$ CI: $3 \cdot 1-5 \cdot 2$ ).

The different patterns of response by age, and the questions this raised about possible bias, stimulated us to look at the relationship of response at phase 2 to that at phase 1 . Table II shows the response to invitation for phase 2 interview in terms of the phase 1 wave at which the

Table II Relationship between acceptance of interview at phase 2 and wave of response at phase 1 : all levels of disability.

\begin{tabular}{lllr}
\hline $\begin{array}{l}\text { Wave of } \\
\text { phase 1 }\end{array}$ & $\begin{array}{l}\text { Number in } \\
\text { phase 2 sample }\end{array}$ & $\begin{array}{l}\text { Number refusing } \\
\text { interview }\end{array}$ & $\left({ }^{\circ}\right)$ \\
\hline 1 & 669 & 25 & $(3 \cdot 7)$ \\
2 & 111 & 8 & $(7 \cdot 2)$ \\
3 & 70 & 6 & $(8 \cdot 6)$ \\
4 & 41 & 14 & $(34 \cdot 1)$ \\
Total & 891 & 53 & $(5 \cdot 9)$ \\
\hline
\end{tabular}


respondent returned the screening questionnaire. Of the 891 eligible and available respondents for the phase 2 interview $6 \%$ refused to participate. Respondents who returned their original questionnaire without reminder were more likely to agree to interview than those responding in the second and third wave. Those responding during the fourth wave follow up were least likely to agree to interview, over one third refusing.

Table II refers to all interviews at all levels of disablement. If only the dependent are considered the proportion refusing was slightly greater, particularly so for those responding during the third and fourth waves (table III). Half of the

Table III Relationship between acceptance of interview at phase 2 and wave of response at phase 1 : respondents with dependence.

\begin{tabular}{lllr}
\hline $\begin{array}{l}\text { Wave of } \\
\text { phase } 1\end{array}$ & $\begin{array}{l}\text { Number in } \\
\text { phase 2 sample }\end{array}$ & $\begin{array}{l}\text { Number refusing } \\
\text { interview }\end{array}$ & $\left({ }^{o}{ }_{o}\right)$ \\
\hline 1 & 417 & 18 & $(4 \cdot 2)$ \\
2 & 65 & 4 & $(6 \cdot 2)$ \\
3 & 35 & 4 & $(11 \cdot 4)$ \\
4 & 26 & 13 & $(50 \cdot 0)$ \\
Total & 543 & 39 & $(7 \cdot 2)$ \\
\hline
\end{tabular}

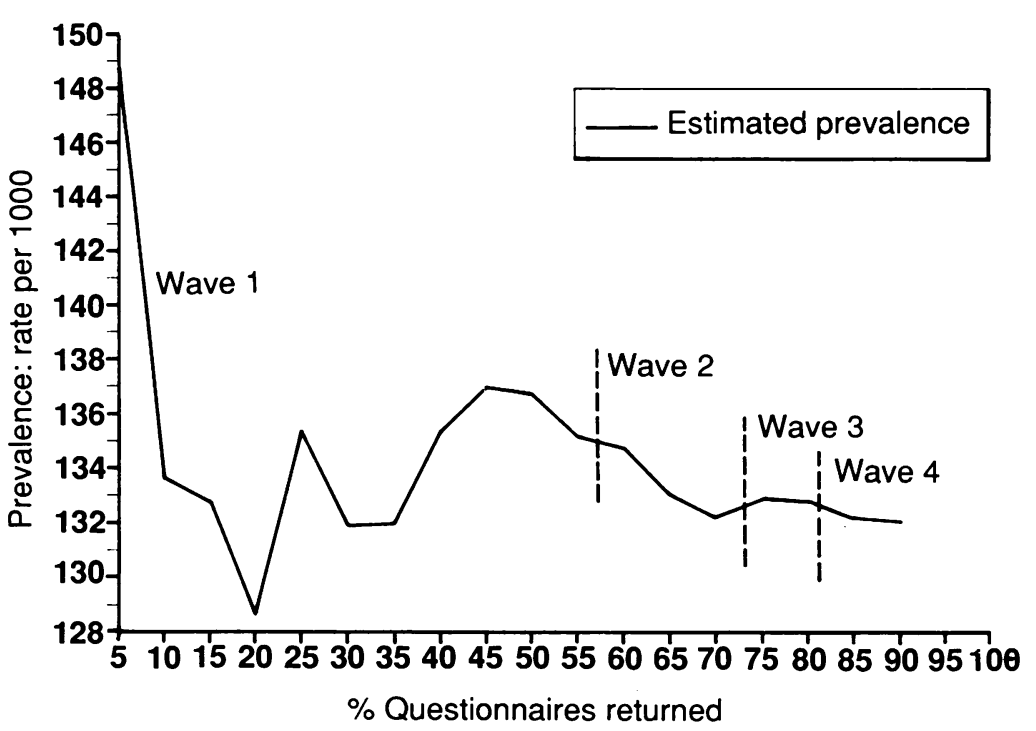

Figure 2 Cumulative rate of dependence (respondents aged $65+$ years)

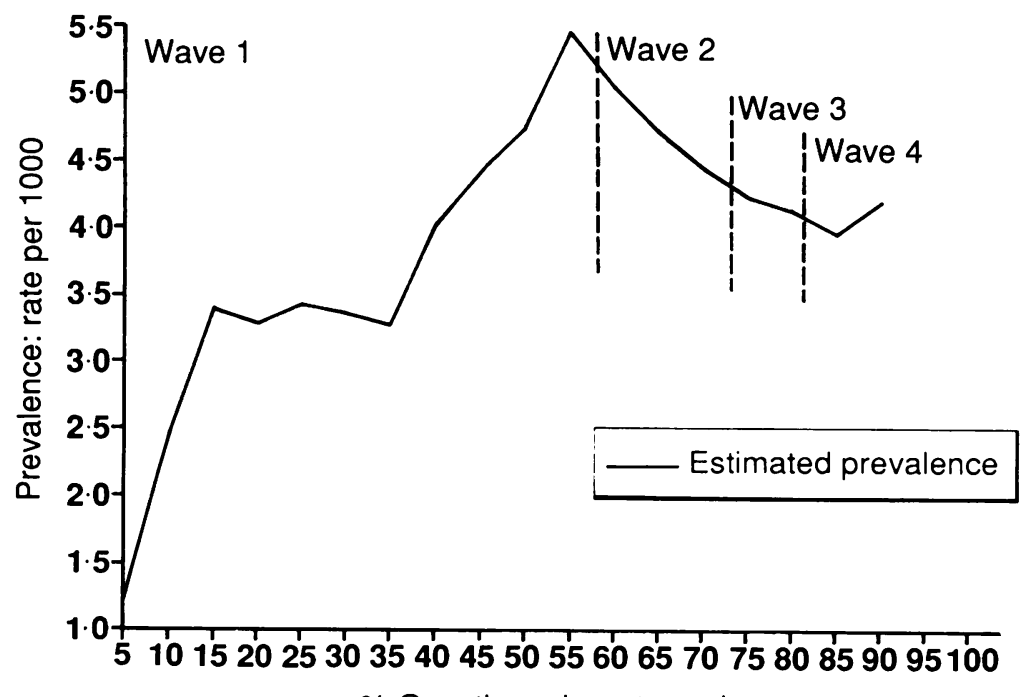

$\%$ Questionnaire returned

Figure 3 Cumulative rate of dependence (respondents aged 16-34 years)
16-64 year old dependent fourth wave respondents refused an interview, including all the available fourth wave 16-34 year olds.

\section{Discussion}

One of two approaches are usually taken when the problem of non-response is considered. The first is a comparison of the characteristics of responders with known population characteristics, for instance, their age and sex. A reasonable match gives confidence that the sample is "representative" of the population under study. This says nothing about the characteristic under investigation, and may lead to unjustifiable confidence about the lack of bias. Ideally there is a need to concentrate on issues of substantive interest rather than on purely demographic traits when examining non-response. ${ }^{9}$ The second approach is to test if late responders differ on key characteristics from those who responded earlier. ${ }^{10-12}$ In general the study of response over time, wave analysis, has provided a useful way of gaining insight into the nature of nonresponse, ${ }^{13} 14$ and this was the approach used in this study.

While the general subject of interest of the survey may have a bearing on the nature of non-response, it is by no means certain even for surveys examining the same subject (eg, community surveys of disability) that nonresponse will be similar. In our survey different response trends for different age groups were revealed by examination of cumulated estimated prevalence across successive waves. Various methods of determining a full coverage prevalence estimate, based on such trends and thus accounting for any bias, have been used. ${ }^{815}$ Evidence from the current survey would suggest a prudent prerequisite for such adjustments would be a close examination of trends among individual age-sex groups, and any other key groups, particularly if disaggregated estimates are required.

The fact that response to the second (interview) phase of the survey was much less in those who reluctantly responded to the first phase raises separate questions about the value of the final phase 1 follow up to increase the response rate. This is of particular concern to us as the effect was more marked among the younger people with dependence. If late responders are a special group and less likely to participate further, it suggests the desirability of a more sophisticated approach to secure a second phase interview, as the small number of interviews arising would be of particular interest. However if the principal target group had been the elderly, where phase 1 prevalence varied little across stages, it is difficult to see how such additional effort and expense would have been worthwhile.

The different patterns of reply across age groups have implications not only for potential biases in the findings, but also for the place given to methodological monitoring in routine survey practice. Examination of age specific response patterns would seem important to be able to identify small but theoretically important groups who appear to be reluctant to respond, and an essential base upon which adjustments to 
accommodate such bias should be considered. Also in a two phase survey, information arising from monitoring could help target additional efforts in the second stage on those groups deemed least likely to participate.

1 Hill AB. Principles of medical statistics: non-response. Lancet 1971; i: 20-2.

2 Newcastle Health Authority and City Council. Disabled and handicapped in the community: 1972-1984. Newcastle: Newcastle City Council, 1985

3 Harris AI. Handicapped and impaired in Great Britain. London: OPCS, 1971

4 Badley EM, Tennant A. Calderdale Health and Disablement Survey. Manchester: Arthritis and Rheumatism Council for Research, 1988.

5 Knight R, Warren M. Physically disabled people living at home: a study of numbers and needs. London: HMSO, 1978.

6 Kalton G, Anderson DW. Sampling rare populations. $\mathcal{F} R$ Stat Soc $A$ 1986; 149: 65-82.
7 Deming WE. An essay on screening, or on two-phase A to surveys of a community. Int Stat Rev 1977; 45: 29-37.

8 Tennant A, Badley EM. A confidence interval approach to investigating non-response bias and monitoring response to postal questionnaires. $\mathcal{F}$ Epidemiol Community Health 1991; 45: $81-5$.

9 Hawkins DF. Estimation of nonresponse bias. Sociol Methods Res 1975; 3: 461-88.

10 Edgerton HA, Britt SH, Normal RD. Objective differences among various types of respondents to a mail questionnaire. Am Sociol Rev 1947; 12: 435-44.

11 Korelitz J, Visscher B. A method for examining the direction and amount of bias due to survey nonresponse (abstract) Am f Epidemiol 1988; 128: 945.

12 Stinchcombe AL, Jones C, Sheatsley P. Nonresponse bia for attitude questions. Public Opinion 1981; 45: 359-75.

13 Ferber $R$. The problem of bias in mail returns: a solution Public Opinion 1948; 12: 669-76.

14 Daniel WW. Nonresponse in sociological surveys: a review of some methods for handling the problem. Sociol Methods Res 1975; 3: 291-307.

15 Locker D, Wiggins R, Sittampalam Y, Patrick DI. Estimating the prevalence of disability in the community: the influence of sample design and response bias. $f$ Epidemiol Community Health 1981; 35: 208-12. 